\title{
ANÁLISE DA VARIABILIDADE DA PRECIPITAÇÃO PARA O ESTADO DE MINAS GERAIS (1981-2017)
}

\author{
PEREIRA, Gabriel - pereira@ufsj.edu.br \\ Universidade Federal de São João del Rei / UFS]
}
CARDOZO, Francielle da Silva - franciellecardozo@ufsj.edu.br Universidade Federal de São João del Rei / UFS]

NEGREIROS, André Batista de - andrebnegreiros@ufsj.edu.br Universidade Federal de São João del Rei / UFS]

ZANIN, Gustavo Domingos - zanin@ufsj.edu.br Universidade Federal de São João del Rei / UFS]

COSTA, Julio Cezar da - julioczcta@gmail.com Universidade Federal de São João del Rei / UFS]
LIMA, Thaínne Eve Ribeiro - thainne.ribeiro@outlook.com Universidade Federal de São João del Rei / UFS]

RUFINO, Paulo Ricardo - pauloricardorufino@gmail.com Universidade Federal de São João del Rei / UFS]

RAMOS, Raquel de Cássia - raquel.cassia15@gmail.com Universidade Federal de São João del Rei / UFS]

\begin{abstract}
RESUMO: A variabilidade da precipitação é de fundamental importância para estudos climatológicos e relacionados ao ciclo hidrológico, e sua concentração afeta consideravelmente as atividades econômicas. Neste contexto, o presente trabalho tem como objetivo principal analisar a variabilidade da precipitação, o início e término da estação chuvosa e sua tendência para o estado de Minas Gerais a partir dos dados de precipitação mensal do produto Climate Hazards Group InfraRed Precipitation with Stations (CHIRPS) para 1981 a 2017. O estado foi agrupado em regiões homogêneas a partir da Análise de Componentes Principais (ACP), que remove a redundância de informações. Os dados da tendência espacial de precipitação foram calculados no aplicativo Grads a partir da função Itrend pelo método dos mínimos quadrados. Por fim, o início e o término da estação chuvosa foi estimado a partir da técnica descrita em Liebmann et al., (2012). Os resultados indicam que o estado de Minas Gerais foi dividido em sete regiões e, de uma forma geral, apresenta uma estação úmida (outubro a março) e uma estação seca (abril a setembro), na qual o mês de janeiro se apresenta como o mais chuvoso e o mês de julho o mês mais seco, sendo que cada uma das sete regiões climáticas do estado exibem características distintas. A análise da variabilidade da duração da estação chuvosa demonstrou que, em geral, a duração média da estação chuvosa é menor nas porções norte de Minas Gerais, aumentando em até 25 dias no extremo sul do estado, sendo que a média da duração da estação chuvosa estimada é de aproximadamente 183 dias. Ainda, a análise da tendência da duração chuvosa indica que a região norte apresenta a tendência de diminuição da estação chuvosa, a região central do estado não apresentou uma tendência na redução ou ampliação da estação chuvosa e a região sul e extremo oeste de Minas Gerais apresenta uma tendência de aumento de dias relacionados à estação chuvosa, denotando a presença de um ciclo hidrológico característico que varia de acordo com a posição.
\end{abstract}


PALAVRAS-CHAVE: Chuva, Variabilidade, Tendência, Regionalização. PRECIPITATION VARIABILITY ANALYSIS FOR MINAS GERAIS STATE (1981-2017)

\begin{abstract}
Precipitation variability is of fundamental importance for climatological studies and related to the hydrological cycle, and its concentration considerably affects economic activities. Thus, the present work has as main objective to analyze the spatial distribution of precipitation, the beginning and the end of the rainy season and its tendency to Minas Gerais state from monthly precipitation data of Climate Hazards Group InfraRed Precipitation with Stations (CHIRPS) product for 1981 to 2017. The state was grouped into homogeneous regions using the Principal Components Analysis (PCA) multivariate statistical technique, which removes the redundancy of information. The precipitation spatial trend data were calculated in Grads software from the Itrend function by least squares method. Finally, the beginning and end of the rainy season was estimated using the technique described in Liebmann et al., (2012). The results indicate that the state of Minas Gerais was divided into seven regions and, in general, presents a humid season (October to March) and a dry season (April to September), where January presents the highest volume of precipitation and July is the driest month, and each one of the seven climatic regions exhibit different characteristics. The rainy season variability analysis showed that, in general, the average duration of the rainy season is lower in the northern portions of Minas Gerais, increasing up to 25 days in the southern end of the state, and the estimate of the average rainy season duration is approximately 183 days. Thus, the analysis of rainfall duration indicates that the northern region presents a tendency to decrease in the rainy season, the central region of the state did not present a tendency in the reduction or expansion of the rainy season and the southern and extreme west region of Minas Gerais presented a trend of increasing days related to the rainy season, denoting the presence of a characteristic hydrological cycle that varies according to the position.
\end{abstract}

KEYWORDS: Rainfall, Variability, Trend, Regions.

\title{
INTRODUÇÃO
}

A precipitação é considerada uma das variáveis mais importantes no ciclo hidrológico. Esta é responsável pela maior parte do input de água na bacia hidrográfica, sendo constantemente utilizada em diversas áreas do conhecimento (PEREIRA et al., 2013). A variação espacial da precipitação no ambiente é ampla e depende de diversos fatores, como a localização geográfica, a altitude, a distância das fontes de umidade, a temperatura e a direção e intensidade dos ventos (BERTONI e TUCCI, 2001).

Diversos fatores podem provocar mudanças no regime de precipitação, dentre eles podem-se citar os desmatamentos, a urbanização desenfreada e a emissão de gases poluentes para a atmosfera, além da intensificação da atividade solar e fenômenos naturais como o El Niño e La Niña (MARENGO, 2010). Assim, a análise espacial da precipitação permite observar as mudanças no comportamento e determinar em quais regiões uma determinada variável vem sofrendo mudanças significativas ao longo do tempo.

Desta forma, conhecer a variabilidade espacial da precipitação em determinada região é de grande importância, pois a modificação dessa variável causa impactos no ciclo hidrológico, nas atividades econômicas e nos elementos do sistema climático. Quando há um excesso de precipitação, o volume maior de chuva pode provocar a alteração no escoamento superficial e subsuperficial, que podem desencadear ou aumentar a erosão e o assoreamento de rios urbanos, gerando inundações. Ainda, o déficit no volume de precipitação pode provocar a ocorrência de secas extremas (TWARDOSZ et al., 2015). Assim, a precipitação é essencial para o gerenciamento dos recursos hídricos e para as atividades 
socioeconômicas, em especial para as atividades que envolvem o setor agrícola (MORAES et al., 2005).

Porém, o conhecimento adequado sobre o regime de precipitação tornase difícil, principalmente devido ao Brasil possuir áreas extensas que não são abrangidas em sua totalidade pelas estações meteorológicas, fatores que influenciam significativamente nas análises ambientais, nos estudos de escoamento superficial, no déficit hídrico e no balanço de energia. Neste contexto, uma forma eficiente de minimizar este problema decorre da extração de informações sobre o volume da precipitação de determinada região a partir do uso de produtos de sensores orbitais, que são constantemente empregados no entendimento do ciclo hidrológico e nos seus efeitos na circulação atmosférica, nas alterações no calor latente e sensível e nas mudanças climáticas globais e regionais (FRANCHITO et al., 2009).

Dentre os produtos utilizados, pode-se citar o Tropical Rainfall Measuring Mission (TRMM), lançado em 1997 com o objetivo de coletar dados sobre a estrutura da precipitação desde o ano de 1997, fornecendo informações em áreas tropicais e subtropicais a cada 3 horas (KUMMEROW et al., 2000). Recentemente, outro produto foi lançado, o Climate Hazards Group InfraRed Precipitation with Stations (CHIRPS), apresentando precipitação diária e mensal continental desde o ano 1981, para a faixa latitudinal entre $50^{\circ} \mathrm{N}$ e $50^{\circ} \mathrm{S}$ (BAYISSA et al., 2017).

Desta forma, o presente trabalho tem como objetivo principal analisar a distribuição espacial da precipitação e sua tendência para o estado de Minas Gerais, além de verificar o início e término da estação chuvosa para as regiões consideradas homogêneas pelo agrupamento dos dados de precipitação mensal do CHIRPS entre 1981 e 2017.

\section{MATERIAL E MÉTODOS}

\section{ÁREA DE ESTUDO}

A área de estudo é representada pelo estado de Minas Gerais, que se encontra na região Sudeste do Brasil, localizado nas coordenadas $14^{\circ} 13^{\prime} 58^{\prime \prime}$ e

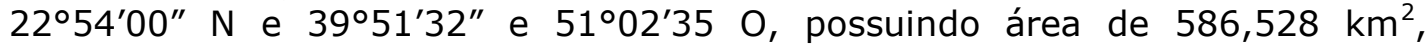
conforme pode ser visualizado na Figura 1. 


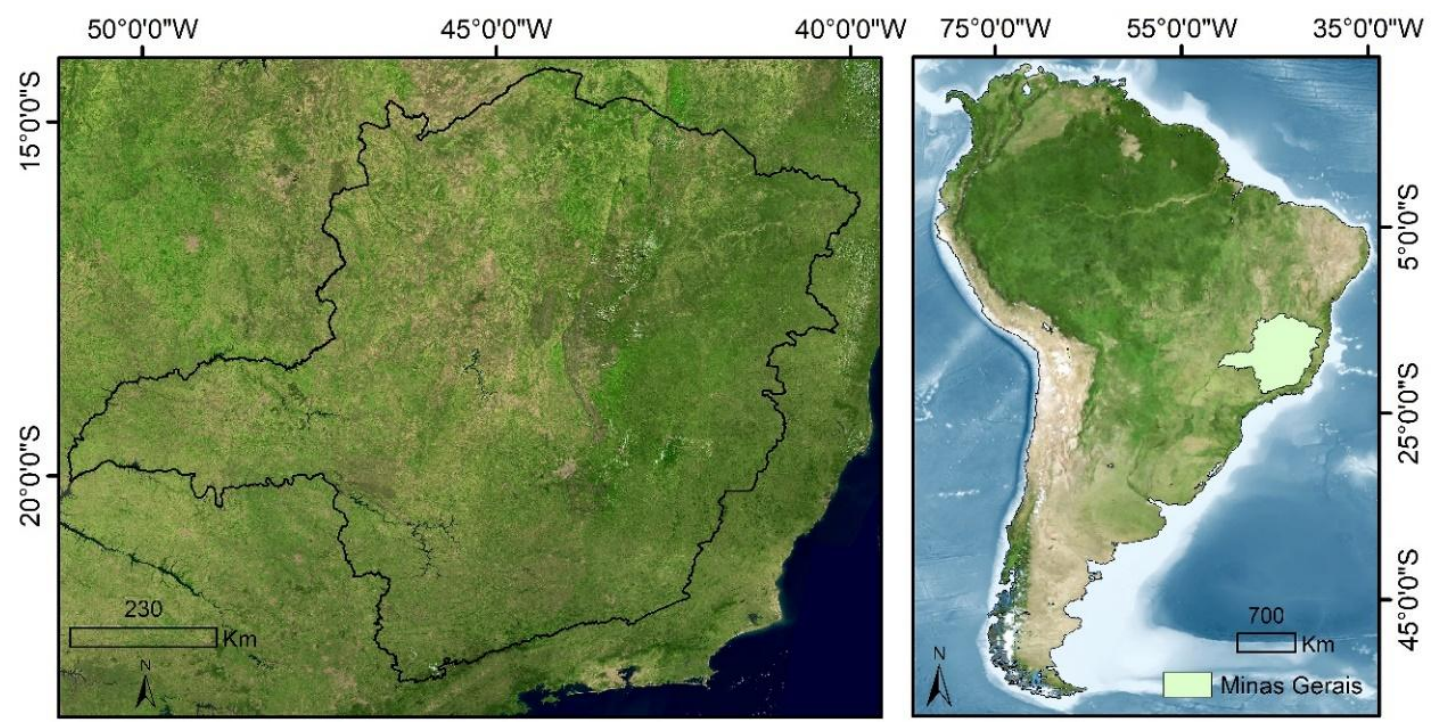

Figura 1 - Localização da área de estudo.

A região Sudeste, onde está inserido o Estado de Minas Gerais, concentra cerca de $42,6 \%$ da população brasileira, assim, as atividades econômicas e a densidade demográfica levam à demanda por abastecimento urbano e geração de energia, tornando a precipitação um elemento de fundamental importância. Localizada próxima ao Trópico de Capricórnio, a região Sudeste apresenta a interferência de vários fenômenos atmosféricos, com destaque para a Zona de Convergência do Atlântico Sul (ZCAS), bem caracterizada no verão (VÁSQUEZ et al., 2018).

De acordo com a classificação de Köppen, o clima em Minas Gerais pode ser dividido em: (a) Clima tropical de savana com estação seca de inverno (Aw), apresentando temperaturas médias acima de $18^{\circ}$ no inverno; (b) Clima subtropical com inverno seco e verão quente (Cwa), possuindo temperaturas médias inferiores a $18^{\circ}$ no inverno e superiores a $22^{\circ}$ no verão; (c) Clima subtropical de altitude com inverno seco e verão ameno (Cwb), apresentando temperaturas médias em torno de $22^{\circ}$; e (d) Clima seco com verão chuvoso (Bsw) (ANTUNES, 1986). A estação chuvosa abrange o período de outubro a março e a estação seca ocorre entre os períodos de abril a setembro, e a precipitação varia de acordo com a posição geográfica e com o relevo. Na região norte ocorrem por volta de $650 \mathrm{~mm}$ de precipitação enquanto que na região sul/sudeste do estado os valores podem atingir $2.100 \mathrm{~mm}$ (GUIMARÃES et al., 2010).

\section{MATERIAIS UTILIZADOS}

\section{CHIRPS}

O conjunto de dados de precipitação Climate Hazards Group InfraRed Precipitation with Stations (CHIRPS) foi desenvolvido pelo United States Geological Survey (USGS) e pelo Climate Hazards Group (CHG) at the University of California, Santa Barbara (UCSB). Nesse produto, as estimativas de 
precipitação são compostas por diversas fontes de informações: (I) Dados de precipitação média acumulada proveniente do The Climate Hazards Group's Precipitation Climatology (CHPClim); (II) Observações de satélites geoestacionários do canal infravermelho termal (Thermal Infrared, TIR) da National Oceanic and Atmospheric Administration (NOAA), produtos do Centro de Previsão Climática (CPC) e o produto B1 do canal TIR proveniente do National Climatic Data Center (NCDC); (III) Campos de Precipitação do Coupled Forecast System da NOAA, versão 2 (CFSv2); (IV) Diversas observações de precipitação através de produtos de estações meteorológicas e outros serviços regionais (FUNK et al., 2015).

O produto CHIRPS possui uma resolução espacial de $0,05^{\circ}$, ou aproximadamente $5,3 \mathrm{~km}$ próximo ao equador, cobertura geográfica de $50^{\circ} \mathrm{S}$ a $50^{\circ} \mathrm{N}$ e é disponibilizado em conjuntos de dados diários, em pêntadas e dados mensais, disponíveis no sítio eletrônico da UCSB (ftp://ftp.chg.ucsb.edu/pub/org/chg/products/CHIRPS-2.0/), em formato NetCDF, GeoTiff e Esri BIL. Diversos estudos validaram os produtos provenientes do CHIRPS, como Nogueira et al., (2018), que avaliaram a eficácia do CHIRPS para a região Sul-Sudeste do estado de Minas Gerais utilizando dados das estações meteorológicas da região obtidas do Instituto Nacional de Meteorologia (INMET). Em geral, os autores mostraram que os dados do CHIRPS apresentaram padrão similar aos dados das estações e, ainda, que os dados foram eficientes na determinação de parâmetros como a deficiência de água, excedente de água e evapotranspiração, mostrando ser uma alternativa na utilização para avaliar dados de precipitação.

\section{PROCEDIMENTOS METODOLÓGICOS}

\section{ANÁLISE POR COMPONENTES PRINCIPAIS}

A Análise de Componentes Principais (ACP), ou Função Ortogonal Empírica (Empirical Orthogonal Function - EOF) é um dos métodos estatísticos mais usados quando se pretende analisar dados multivariados e fazer correlações entre dados multitemporais. Geralmente, os dados que compõem uma série histórica ou diversas bandas de um mesmo sensor são correlacionadas (visualmente e numericamente similares), assim, a análise individual de cada informação pode não ser tão eficaz devido à informação redundante presente. Desta forma, a ACP é uma técnica matemática de realce que reduz ou remove a redundância de informações e geram um novo conjunto de dados cujas bandas individuais originadas no processo apresentam informações não disponíveis em outras camadas de informação.

Para a aplicação da técnica, é utilizado um conjunto de $\mathrm{N}$ imagens de entrada, que depois de processadas pela $A C P$, vai produzir um outro conjunto de $\mathrm{N}$ imagens de saída com nenhuma correlação entre si. O primeiro conjunto de saída é chamado de primeira componente principal, ou CP1, o segundo conjunto de saída é chamado de CP2, o terceiro, de CP3, e assim por diante. Desta forma, a CP1 irá conter a informação que é comum a todas as $\mathrm{N}$ bandas originais, a CP2 conterá a feição mais significante do conjunto, as componentes seguintes conterão feições espectrais cada vez menos significantes até sobrar a última $\mathrm{CP}$, que conterá a informação menos significante (CROSTA, 1992). A vantagem do uso dessa técnica decorre da remoção da redundância das informações. Neste caso, os dados podem ser descritos de uma forma mais 
concisa e certas características escondidas na série temporal podem ser realçadas por esta técnica de estatística multivariada.

\section{PROCESSO DE AGRUPAMENTO E TENDÊNCIA}

A análise de clusters, ou agrupamento, é utilizada para separar dados em grupos distintos, que serão determinados a partir dos parâmetros estatísticos relacionados a partir do grau de similaridade entre as observações individuais (WILKS, 2011). A análise de clusters utiliza o conceito de distância entre os elementos que compõem o conjunto de dados. Desta forma, as dez primeiras CP geradas a partir dos dados de precipitação do CHIRPS foram submetidas ao processo de agrupamento, na qual o processo ocorre somente em regiões adjacentes, a partir do critério de similaridade e de agregação adotado. Neste processo de agrupamento, duas regiões são consideradas similares quando a distância euclidiana entre os valores médios ponderados das regiões (D) satisfaz os critérios de similaridade, conforme equação 1.

$$
D_{(r 1, r 2)}=\sqrt{\sum_{n=1}^{N} P_{n}\left(V_{i, k-} V_{j, k}\right)^{2}}
$$

em que r1 e r2 representam duas regiões diferentes na qual a distância euclidiana será calculada, $\mathrm{P}$ é o peso aplicado aos dados, $\mathrm{N}$ representa a dimensionalidade dos dados vetoriais, Vi e Vj são os vetores.

Ainda, os dados de precipitação para o estado de Minas Gerais foram inseridos no aplicativo Grads. Neste, utilizou-se a função denominada Itrend para calcular a tendência espacial da série temporal de precipitação a partir do método dos mínimos quadrados. A tendência é calculada minimizando-se a soma dos quadrados da diferença, otimizando o ajuste do modelo aos dados de precipitação (xi), conforme equação 2 .

$$
S Q=\sum_{i=1}^{n}\left(Y_{i}-b-a x_{i}\right)^{2}
$$

em que SQ representa a soma dos quadrados dos desvios, Y_i é o valor previsto, a é o coeficiente angular da equação de regressão linear, b é a constante da equação.

\section{DETERMINAÇÃo DO INÍCIO, TÉRMINO E DURAÇÃo DO PERÍODO DE PRECIPITAÇÃO}

Para estimar o início e o término da estação chuvosa utilizou-se a técnica descrita em Liebmann et al., (2012), que consiste na comparação da precipitação média diária para cada um dos dias do ano (Qi) com a precipitação diária acumulada (Q). Este método consiste em estimar a anomalia de precipitação diária cumulativa $\mathrm{C}(\mathrm{d})$ para determinado dia (d), conforme Equação 3:

$$
C(d)=\sum_{i=1}^{d} Q i-\bar{Q}
$$

em que i representa os dias do ano. Ressalta-se que o dia do mínimo valor em C marca o início da estação chuvosa e o valor máximo o final da 
estação chuvosa. Para o processamento de $\mathrm{C}(\mathrm{d})$ os dados do CHIRPS foram inseridos no aplicativo Grads e uma máscara espacial com as regiões obtidas do agrupamento foram utilizadas para calcular a precipitação média diária para cada região. Após a aplicação da máscara e da média para a área, os dados foram exportados em formato binário e lidos a partir de um algoritmo em IDL, com o objetivo de estimar para cada ano o início e término da estação chuvosa a partir do ajuste de uma função polinomial sobre os dados.

\section{RESULTADOS E DISCUSSÃO}

$\mathrm{Na}$ literatura é possível encontrar diversos estudos relacionados à aplicabilidade da técnica de análise por componentes principais e variáveis meteorológicas. Wilson-Diaz et al., (2001) utilizaram a ACP para comparar a variabilidade da temperatura da superfície do mar (TSM) estimada pelo satélite Advanced Very High Resolution Radiometer (AVHRR) e dados de modelos numéricos sobre o oceano Índico, indicando que a primeira componente compreendia de 58 a $95 \%$ de toda a variabilidade da TSM, associada aos mecanismos de aquecimento e resfriamento ocasionados pelas Monções do Oceano Índico.

Outras pesquisas, como Bordoni e Stevens (2005) utilizaram esta técnica para analisar a variabilidade dos ventos no Golfo da Califórnia a partir dos dados do satélite Quick Scatterometer (QuikSCAT), associando as variações do vento à padrões de larga escala induzidos pela zona de convergência intertropical (ZCIT). Ainda, diversos estudos relacionam a ACP e a precipitação, por exemplo, Teo et al., (2011) indicam que esta técnica é capaz de diagnosticar o ciclo diurno da precipitação na região marítima da Ásia, na qual a primeira componente apresenta as variações da instabilidade atmosférica, originadas dos fluxos radiativos da superfície. Outros estudos utilizam a ACP espacialmente com o objetivo de analisar a variabilidade espacial e temporal da precipitação e sua relação com o planejamento e gerenciamento de recursos hídricos (AMIRI et al., 2017).

Assim, a Figura 2 exibe o resultado da aplicação da ACP nos dados de precipitação do CHIRPS para a série temporal de 01 de janeiro de 1981 a 31 de dezembro de 2017. Neste trabalho, a ACP foi utilizada para analisar os padrões de distribuição da precipitação no estado de Minas Gerais associados à correlação espacial destas informações e suas variações multitemporais. Desta forma, ao decompor a série dos dados do CHIRPS em componentes, optou-se por utilizar as 10 primeiras componentes, pois estas contêm aproximadamente $99 \%$ da variabilidade dos dados (CRADDOCK, 1965). A Figura 2a exibe uma composição colorida das três primeiras componentes, na qual a composição escolhida foi 1B2G3R. 


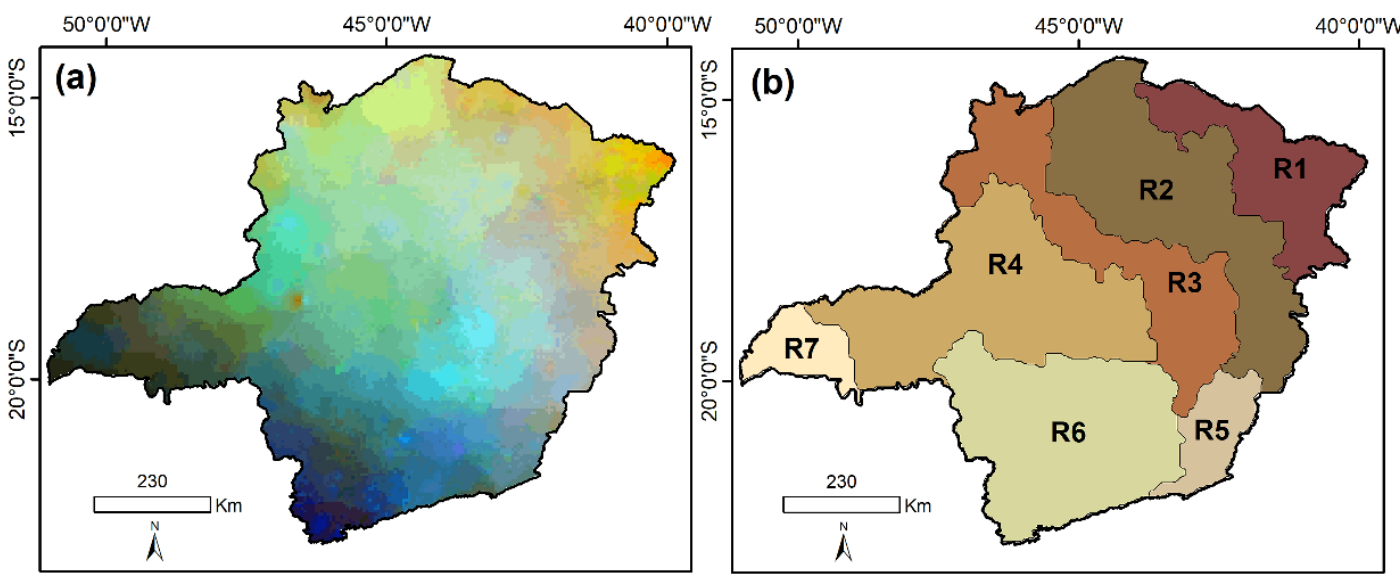

Figura 2 - Composição colorida das três primeiras componentes da ACP (a); resultado da clusterização das dez primeiras componentes, dividindo o estado de Minas Gerais em 7 regiões diferentes (b).

Embora visualmente perceba-se uma diferença entre diversas áreas do estado de Minas Gerais (Figura 2a), optou-se por utilizar a técnica estatística de agrupamento de regiões, ou cluster, para separar as regiões do estado reduzindo, desta forma, a redundância de informações. O resultado do agrupamento e separação das áreas pode ser visualizado na Figura 2b. Assim, para Minas Gerais, a integração da ACP e da técnica de agrupamento permitiu separar o estado em 7 regiões distintas, que receberam a nomenclatura no sentido Norte-Sul, sendo a primeira região denominada de R1 e a última de R7.

O estado de Minas Gerais, devido à sua localização geográfica, possui características de um clima de transição, desta forma, atuam na região fenômenos meteorológicos de latitudes médias, temperadas e tropicais. No inverno, a região sofre a influência de frentes frias, que ocasionam áreas de instabilidades e geralmente vem seguidas de uma massa de ar frio proveniente do anticiclone migratório polar, que provoca queda das temperaturas do ar (ABREU, 1998). Ainda, no inverno a atuação da Alta Subtropical do Atlântico Sul (ASAS), caracterizada por forte subsidência, impede a formação de áreas de instabilidade, criando condições desfavoráveis para a precipitação, ou seja, propiciando uma estação seca bem definida.

No verão, o desenvolvimento de instabilidades atmosféricas a partir do aquecimento da superfície terrestre e fenômenos como a Zona de Convergência do Atlântico Sul (ZCAS) ocasiona grande volume de chuva. Neste contexto, a Figura 3a mostra o gráfico da Região 1, composta pelas porções Norte de Minas Gerais, Jequitinhonha e Vale do Mucuri, e descreve a variabilidade da precipitação mensal obtida pelos dados do CHIRPS de 01 de janeiro de 1981 a 31 de dezembro de 2017. A Figura 3a exibe um diagrama com informações da distribuição estatística do conjunto de dados, composto pela mediana (parâmetro relacionado à tendência central do conjunto de dados), quartil inferior e superior (que compreendem $50 \%$ de todos os valores mensais), os valores máximos e mínimos (com exceção dos outliers) e os outliers (representados pelos círculos). 

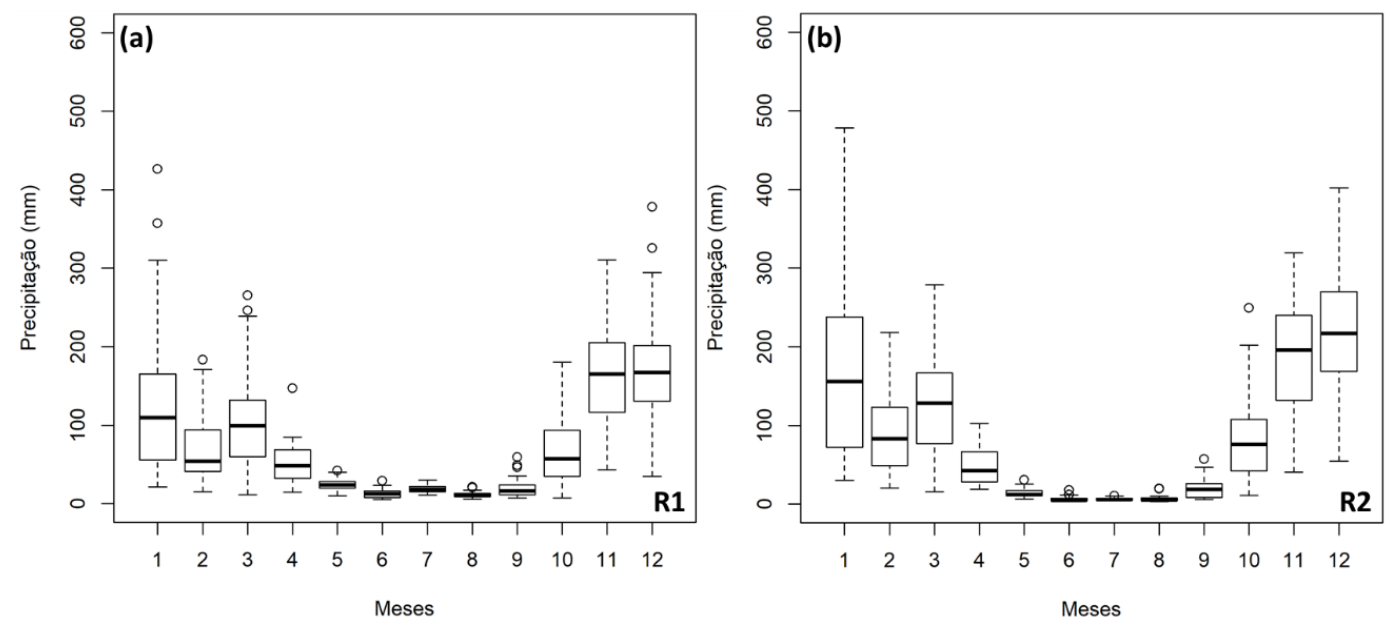

Figura 3 - Gráfico boxplot da precipitação estimada pelo produto CHIRPS (1981 a 2017) para os meses de janeiro a dezembro para a região 1 (a) e região 2 (b).

Ao analisar o gráfico da Figura $3 a$, verifica-se que a Região 1 (R1) apresentou a mesma característica da variabilidade da precipitação existente para o estado: verões chuvosos e o período seco coincidindo com o inverno, característico do clima tropical úmido de savana (cerrado) na qual a região está inserida. Ainda, verificou-se que essa região registra os menores valores de precipitação em relação às demais áreas de Minas Gerais, sendo que dezembro pode ser considerado o mês com maior volume de precipitação. Entretanto, janeiro apresenta o maior volume mensal absoluto, aproximadamente $450 \mathrm{~mm}$. Para a Região 1, os meses de junho e agosto são os meses mais secos, característica ocasionada pelo domínio de situações anticiclônicas que impossibilitam o avanço de sistemas mais úmidos (NUNES et al., 2009).

A Figura 3b mostra o gráfico da Região 2 (R2), que compreende grande parte do Norte de Minas Gerais (porção mais ao Sul), Vale do Jequitinhonha e Vale do Rio Doce. Nesta região nota-se que o mês de fevereiro apresentou o menor volume de precipitação durante a estação chuvosa, similar ao que acontece na Região 1, com precipitação máxima de aproximadamente 200 mm, tendência verificada em toda a área de estudo devido à influência da Zona de Convergência do Atlântico-Sul (ZCAS), que desloca a precipitação para o Sul do estado (PERTUSSATTI, 2013; INMET, 2017).

Ainda, é verificado nessa região uma sazonalidade tipicamente tropical com dois períodos bem caracterizados, onde os totais pluviométricos são maiores no período entre outubro e março, que registram também as maiores temperaturas. Entretanto, a sazonalidade climática nessa região não se apresenta de forma uniforme e regular. O mês de janeiro foi o que apresentou a maior variabilidade pluviométrica (entre 30 e $480 \mathrm{~mm}$ ), contrastando com o mês de fevereiro, em que a variabilidade é muito menor (entre 20 e 180mm). A oscilação nos meses chuvosos deriva de fenômenos como o veranico (ocasionado por bloqueios atmosféricos) e por outros elementos associados aos anos de ocorrência de El Niño e La Nina, um dos principais mecanismos responsáveis pela variabilidade das precipitações pluviais durante o período chuvoso em Minas Gerais (MINUZZI, 2005). 
Na análise da variabilidade da precipitação para as áreas originadas no agrupamento das componentes principais, a Região 3 (R3) apresenta pluviosidade mais significativa quando comparada com as regiões 1 e 2 . Estabelecida em partes do Noroeste de Minas Gerais, Norte, Centro e Região Metropolitana de Belo Horizonte (Figura 4a), observa-se que os maiores valores pluviométricos se concentram no mês de dezembro. Nesta região nota-se a influência do fenômeno veranico, caracterizado pela ausência de precipitação (< $5 \mathrm{~mm}$ ) por dias consecutivos, caracterizado por uma área de estabilidade no verão que diminui a precipitação. A ausência de precipitação pode ser verificada pelos valores mínimos de precipitação nos meses chuvosos, caracterizado pelo quartil inferior.
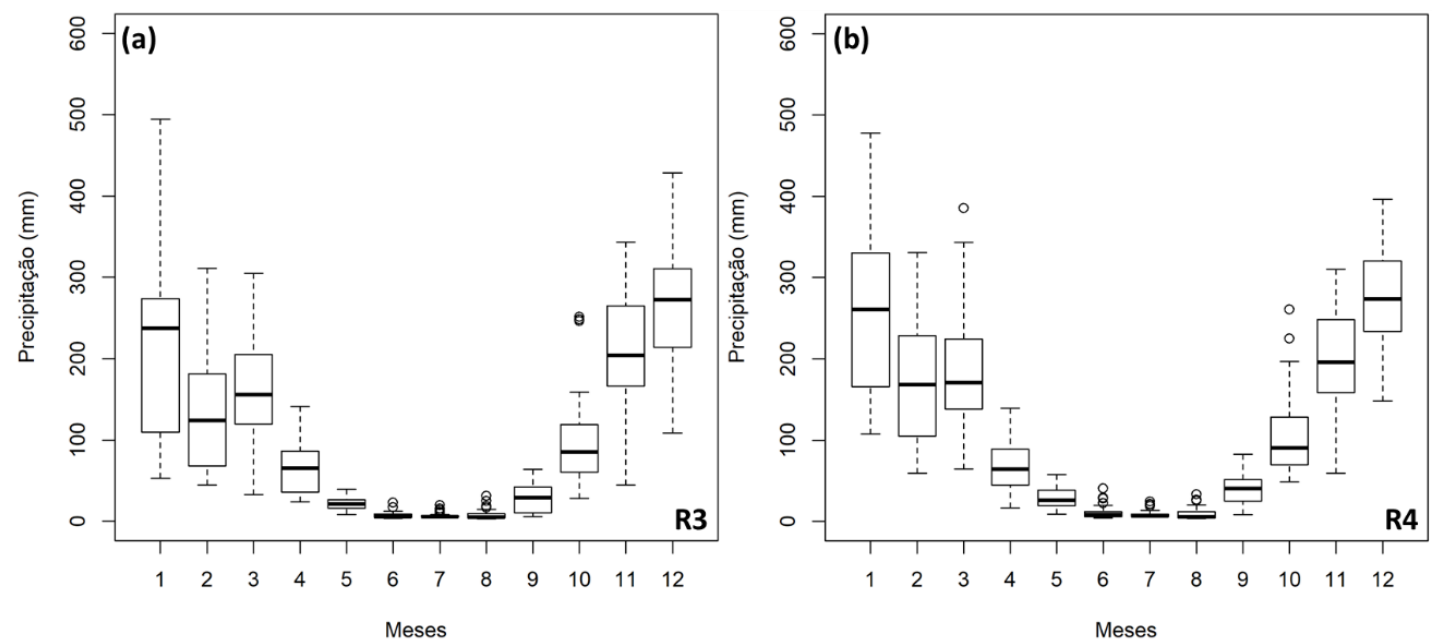

Figura 4 - Gráfico boxplot da precipitação estimada pelo produto CHIRPS (1981 a 2017) para os meses de janeiro a dezembro para a região 3 (a) e região 4 (b).

O gráfico da Região 4 (R4) corresponde à grande parte do Triângulo Mineiro/Alto Paranaíba, Noroeste e Centro de Minas Gerais (Figura 4b). Nesta região, os maiores valores de precipitação se concentraram entre os meses de dezembro e janeiro. Entretanto, os meses que apresentam a maior variabilidade são março e fevereiro. O período chuvoso dessa região sofre influência da topografia, ocasionando chuvas orográficas. Além disso, outro modificador do tempo e clima nessa região é a área de instabilidade ocasionada pela passagem do anticiclone migratório polar, ou depressões frontais (NUNES et al., 2009). Para a Região 4, a estação seca apresentou baixos valores de precipitação, menores que $50 \mathrm{~mm}$ mensais, decorrentes da estabilidade ocasionada pela ASAS.

Ao analisar o gráfico da Região 5, correspondente à Mesorregião da Zona da Mata (Figura 5a), percebeu-se que o mês de janeiro apresentou a maior variabilidade pluviométrica (entre 80 e $490 \mathrm{~mm}$ ), seguido dos meses de novembro ( 80 e $350 \mathrm{~mm}$ ) e dezembro (150 e $480 \mathrm{~mm}$ ). Ademais, os meses de fevereiro, novembro e dezembro apresentaram uma variabilidade semelhante. Assim como as regiões observadas anteriormente, o período chuvoso sofre influência da precipitação convectiva e da ZCAS (NUNES et al., 2009). Nos 
meses da estação seca, as instabilidades sofrem influência dos eventos em escala sinótica e regionais, como, por exemplo, bloqueios atmosféricos e predominância de massas de ar estáveis, quentes e secas (AMBRIZZI et al., 2009).
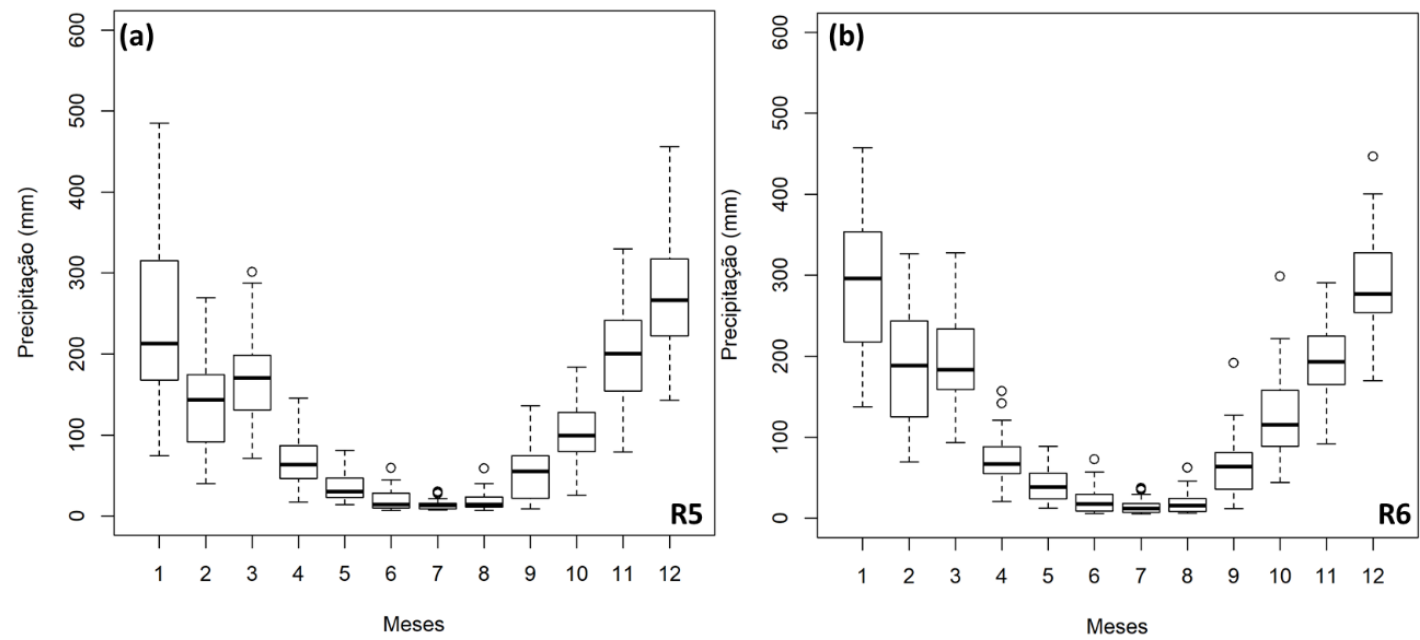

Figura 5 - Gráfico boxplot da precipitação estimada pelo produto CHIRPS (1981 a 2017) para os meses de janeiro a dezembro para a região 5 (a) e região 6 (b).

O gráfico da distribuição da precipitação da Região 6 pode ser visualizado na Figura 5b, que abrange as mesorregiões mineiras Sul e Sudoeste de Minas Gerais, Campo das Vertentes, Oeste de Minas Gerais, região Metropolitana de Belo Horizonte e Zona da Mata. Nesta região, o período com os índices mais elevados alcança médias mensais de 200 a 300 mm, com anos em que a precipitação mensal pode ultrapassar $450 \mathrm{~mm}$. Entretanto, a estação seca é caracterizada por baixos valores pluviométricos, que em alguns anos pode ser caracterizado pela ausência de precipitação. Nesta região não é incomum a ausência de precipitação por mais de 30 dias consecutivos, decorrentes de bloqueios atmosféricos e forte atuação da ASAS, além de altos índices diários e mensais decorrentes da ZCAS (DE MELLO et al., 2013; REBOITA et al., 2015).

A Região 7 é composta unicamente pelo Triângulo Mineiro e Alto Paranaíba. Essa região apresenta características de sazonalidade da precipitação semelhantes às demais regiões (Figura 6a). A estação chuvosa apresenta grande variabilidade entre as médias mensais, com índices que variam entre 80 a $450 \mathrm{~mm}$, com janeiro o mês com maior acumulado de precipitação. O período de seca se caracteriza por meses com pouca precipitação, similar à Região 6, na qual julho é o mês mais seco, caracterizado pelo período de transição do outono/inverno. Assim, o padrão de distribuição de precipitação na área de estudo abrange um período úmido que engloba os meses de outubro a março e um período seco que abrange os meses de abril a setembro. Ainda, os maiores volumes de precipitação são verificados no mês de janeiro, e esses mesmo padrão de distribuição da precipitação no estado de Minas Gerais foi verificado por Mello et al., (2003) e Silva e Reboita (2013). 
A Figura $6 \mathrm{~b}$ exibe um exemplo do gráfico do início e término da estação chuvosa da região 7 para o ano de 2016. Nesta, é possível observar a frequência acumulada da precipitação (eixo y, esquerdo, linha verde), estimada a partir do somatório da anomalia de precipitação diária em relação à média diária do dado anual. Ainda, é possível visualizar a polinômio ajustado à curva dos dados (linha preta) e a definição do início (círculo azul) e término (círculo vermelho) da estação chuvosa. A precipitação diária é visualizada a partir das colunas em azul e seus valores no eixo y (direita). Para cada região, a análise da estação chuvosa foi ponderada pelo ciclo hidrológico, ou seja, os anos foram analisados de acordo com seus ciclos, iniciando no dia 01 de julho e finalizando no dia 30 de junho do ano seguinte.
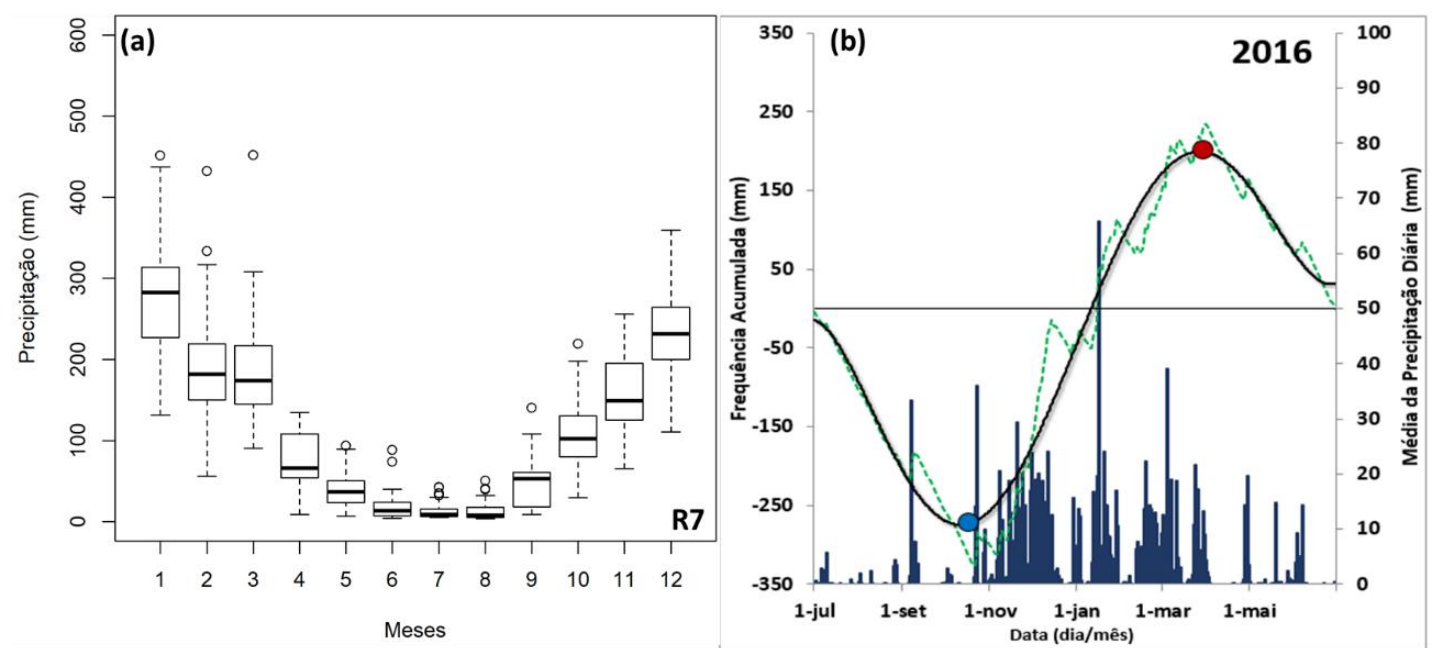

Figura 6 - Gráfico boxplot da precipitação estimada pelo produto CHIRPS (1981 a 2017) para os meses de janeiro a dezembro para a região 7 (a) e exemplo da frequência acumulada da precipitação e a definição do início (em azul) e término (em vermelho) da estação chuvosa da região 7 para o ano de 2016 (b).

A Figura 7 exibe a variabilidade da duração da estação chuvosa para o período compreendido entre 1981 a 2017, referente às regiões originadas pelo processo de agrupamento da ACP. Para cada ano hidrológico (de 01 de julho a 30 de junho), que representa a estação chuvosa, a equação polinomial foi estimada automaticamente para todas as regiões. Ao comparar a amplitude inferior e a superior, estimou-se o tempo de duração entre o início da estação e o final da mesma. Em geral, a duração média da estação chuvosa é menor nas porções norte de Minas Gerais, aumentando em até 25 dias no extremo sul do estado. A média da duração da estação chuvosa estimada para Minas Gerais é de aproximadamente 183 dias, com as regiões 1 e 2 apresentando as menores médias (176 e 178 dias, respectivamente) e as regiões 5 e 6 com a maior duração (189 e 193 dias, respectivamente). 

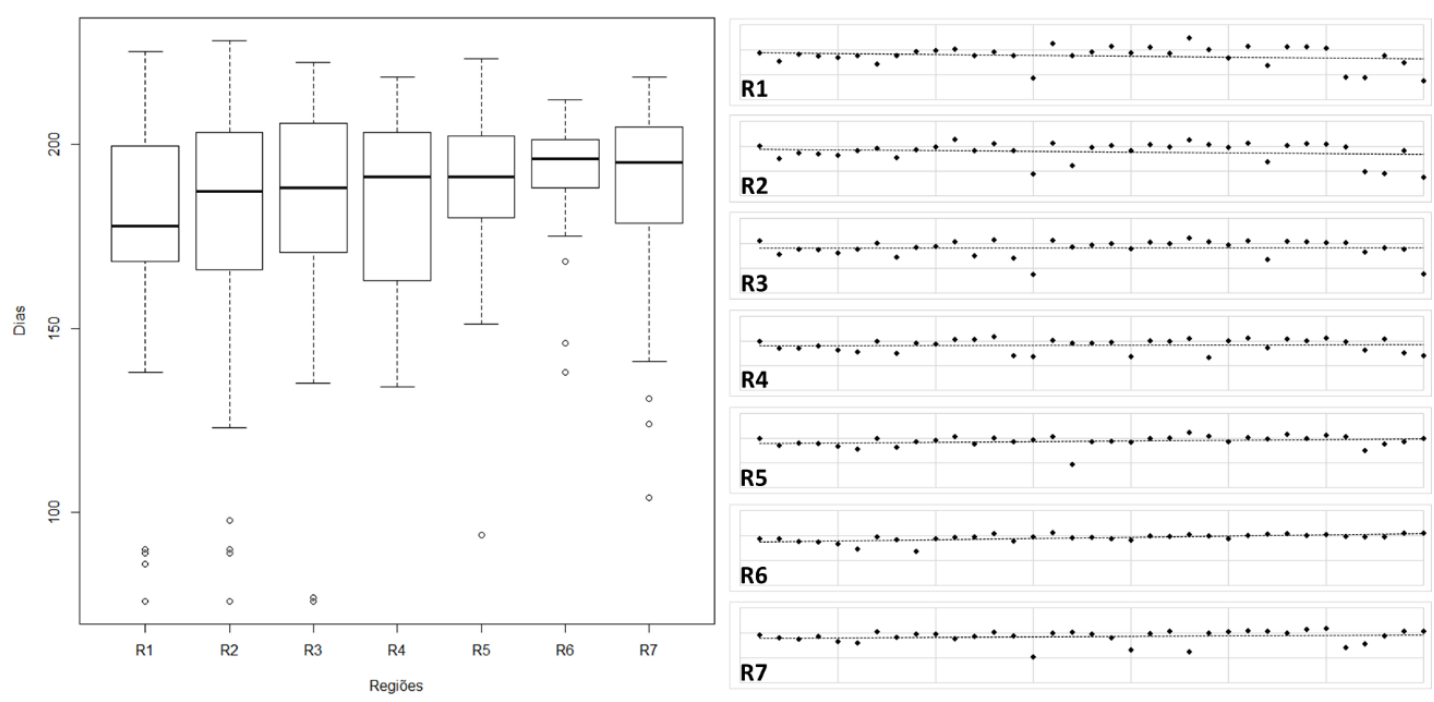

Figura 7 - Gráfico boxplot da duração da estação chuvosa para as regiões de Minas Gerais.

Das regiões analisadas, a região 6, inserida nas mesorregiões mineiras Sul e Sudoeste, Campo das Vertentes, Oeste de Minas, Metropolitana de Belo Horizonte e Zona da Mata, apresenta a menor variabilidade, ou seja, seu ciclo hidrológico apresenta poucas variações no decorrer de 35 anos, com exceção de anos atípicos de baixa precipitação na estação chuvosa (3 casos). Porém, tal característica difere das demais regiões, uma vez que as regiões Norte e Sul de Minas Gerais apresentaram as maiores variabilidades, nestas, a duração da estação chuvosa pode variar de 140 a 230 dias, indicando que fenômenos de bloqueio atmosférico e estabilidade atmosférica afetam regularmente o volume de precipitação e a umidade relativa do ar.

A análise da tendência da duração chuvosa, visualizada nos gráficos da Figura 7, na qual a linha pontilhada representa a tendência positiva ou negativa dos dados e os pontos os valores da duração da estação chuvosa por ano (1981 a 2017), indica que as regiões 1 e 2 apresentam a tendência de diminuição da estação chuvosa ( $p<0.10$, teste t-student), enquanto que as regiões 3,4 e 5 não apresentaram uma tendência na redução ou ampliação da estação chuvosa. Entretanto, as regiões 6 e 7 apresentaram uma tendência de aumento de dias relacionados à estação chuvosa $(p<0.05$, teste t-student). Ao verificar a tendência espacial para a precipitação do estado de Minas Gerais (não exibido), percebe-se uma tendência de aumento nas regiões mencionadas relacionada com a ampliação da estação chuvosa. Desta forma, percebe-se que Minas Gerais é composta por um ciclo hidrológico característico, que varia de acordo com a posição no estado, com valores mais elevados ao sul e características mais secas no norte do estado.

\section{CONCLUSÕES}

Neste trabalho foi apresentado a variabilidade da precipitação para o estado de Minas Gerais nos anos de 1981 a 2017, utilizando a técnica estatística de agrupamento de regiões para separar o estado em sete regiões que apresentavam características homogêneas. De uma forma geral, percebe-se que 
a área de estudo apresenta uma estação úmida que abrange os meses de outubro a março e uma estação seca no inverno, que abrange os meses de abril a setembro, sendo o mês de janeiro o mais chuvoso e o mês de julho o mais seco de todo o estado.

Em relação às sete regiões climáticas do estado, as mesmas exibem algumas particularidades. A Região 1 (R1 - Norte de Minas Gerais, Jequitinhonha e Vale do Mucuri) é a região mais seca e com os menores registros de precipitação de todo o estado; a Região 2 (R2 - grande parte do Norte de Minas Gerais, Vale do Jequitinhonha e Vale do Rio Doce) possui uma sazonalidade tipicamente tropical com dois períodos bem caracterizados, porém a variabilidade climática não se apresenta de forma uniforme e regular; a Região 3 (R3 - partes do Noroeste de Minas Gerais, Norte, Centro e Região Metropolitana de Belo Horizonte) caracteriza-se pela ausência de precipitação por vários dias consecutivos devido à atuação do fenômeno veranico; na Região 4 (R4 - grande parte do Triângulo Mineiro/Alto Paranaíba, Noroeste e Centro de Minas Gerais) o volume de precipitação sofre influência da topografia, que ocasiona chuvas orográficas; a Região 5 (R5 - Mesorregião da Zona da Mata) apresenta uma grande variabilidade pluviométrica no verão; na Região 6 (R6 mesorregiões mineiras Sul e Sudoeste de Minas Gerais, Campo das Vertentes, Oeste de Minas Gerais, região Metropolitana de Belo Horizonte e Zona da Mata) a estação seca é caracterizada por baixos valores pluviométricos, que em alguns anos possuem ausência quase total da precipitação por vários meses decorrentes de bloqueios atmosféricos e forte atuação da ASAS; e a Região 7 (R7 - Triângulo Mineiro e Alto Paranaíba) apresenta características de sazonalidade da precipitação semelhantes às demais regiões.

Ainda, a análise da variabilidade da duração da estação chuvosa demonstrou que, em geral, a duração média da estação chuvosa é menor nas porções norte de Minas Gerais, aumentando em até 25 dias no extremo sul do estado, sendo que a média da duração da estação chuvosa estimada para Minas Gerais é de aproximadamente 183 dias, com as regiões 1 e 2 apresentando as menores médias (176 e 178 dias, respectivamente) e as regiões 5 e 6 com a maior duração (189 e 193 dias, respectivamente). Ainda, na região 6 a variabilidade da precipitação apresenta poucas variações no decorrer de 35 anos, com exceção de 3 casos atípicos de baixa precipitação na estação chuvosa, característica que difere das demais regiões, já que as regiões norte e sul de Minas Gerais apresentaram as maiores variabilidades, onde a duração da estação chuvosa pode variar de 140 a 230 dias, indicando que fenômenos de bloqueio atmosférico e estabilidade atmosférica afetam regularmente o volume de precipitação e a umidade relativa do ar.

Por fim, a análise da tendência da duração chuvosa indica que a região norte apresenta a tendência de diminuição da estação chuvosa; a região central do estado não apresenta uma tendência na redução ou ampliação da estação chuvosa e a região sul e extremo oeste de Minas Gerais apresenta uma tendência de aumento de dias relacionados à estação chuvosa, denotando a presença de um ciclo hidrológico característico que varia de acordo com a posição, com valores mais elevados ao sul e características mais secas no norte. Portanto, os resultados obtidos permitem a observação das mudanças que o regime de precipitação vem sofrendo ao longo do tempo na área de estudo, fomentando ações essenciais para o gerenciamento dos recursos hídricos e para as atividades socioeconômicas. 


\section{REFERÊNCIAS BIBLIOGRÁFICAS}

ABREU, M. L. Climatologia da estação chuvosa de Minas Gerais: De Nimer (1977) à Zona de Convergência do Atlântico-Sul, Geonomos, v. 6, n. 2, p.17-22, 1998.

AMBRIZZI, T.; MARQUES, R.; NASCIMENTO, D. H. Clima da Região Sudeste do Brasil. In: CAVALCANTI, I. F. A.; FERREIRA, N. J; SILVA, M. G. A. J.; DIAS, M. A. F. da S. Tempo e clima no Brasil. Oficina de textos, 2009.

AMIRI, A. M.; MESGARI, M. S. Modeling the Spatial and Temporal Variability of Precipitation in Northwest Iran, Atmosphere, v. 8, p. 254, 2017.

ANTUNES, F. Z. Caracterização climática do estado de Minas Gerais: climatologia agrícola, Informe Agropecuário, v. 12, p. 9-13, 1986.

BAYISSA, Y.; TADESSE, T.; DEMISSE, G.; SHIFERAW, A. Evaluation of SatelliteBased Rainfall Estimates and Application to Monitor Meteorological Drought for the Upper Blue Nile Basin, Ethiopia, Remote Sensing, v. 9, n. 7, p. 669, 2017.

BERTONI, J. C.; TUCCI, C. E. Precipitação. In: TUCCI, C. E. M. (Org.). Hidrologia: ciência e aplicação. 2.ed. Porto Alegre: ABRH/Editora UFRGS, 2001, p.177-231.

BORDONI, S.; STEVENS, B. Principal Component Analysis of the Summertime Winds over the Gulf of California: A Gulf Surge Index, Mon. Wea. Rev., v. 134, p. 3395-3414, https://doi.org/10.1175/MWR3253.1, 2006.

CRADDOCK, J. M. A Meterological Application of Principal Component Analysis, Journal of the Royal Statistical Society. Series D (The Statistician), v. 15, n. 2, p. 143-156. www.jstor.org/stable/2987388, 1965.

CROSTA, A. P. Processamento Digital de Imagens de Sensoriamento Remoto. Campinas - SP: IG /UNICAMP, 1992. 170p.

DE MELLO, C. R.; RIBEIRO VIOLA, M. Mapeamento de chuvas intensas no estado de Minas Gerais, Revista Brasileira de Ciência do Solo, v. 37, n. 1, 2013.

FRANCHITO, S. H.; RAO, V. B.; VASQUES, A. C.; SANTO, C. M. E. ; CONFORTE, J. C. Validation of TRMM precipitation radar monthly rainfall estimates over Brazil, Journal of Geophysical Research, v. 114, n. D2, p. 1-9, 2009.

FUNK, C. PETERSON, P.; LANDSFELD, M.; PEDREROS, D.; VERDIN, J.; SHUKLA, S; HUSAK, G.; ROWLAND, J.; HARRISON, L.; HOELL, A.; MICHAELSEN, J. The climate hazards infrared precipitation with stations-a new environmental record for monitoring extremes, Scientific data, v. 2, p. 150066, 2015.

GUIMARÃES, D. P.; REIS, R. J.; LANDAU, E. C. Índices pluviométricos em Minas Gerais. Sete Lagoas: Embrapa Milho e Sorgo, 2010.

INMET - Instituto Nacional de Meteorologia. Nota técnica: 004/17 - Estação chuvosa em Minas Gerais. Disponível em: http://www.inmet.gov.br/portal/notas_tecnicas/2017/OUTUBRO/Nota_tecnica_C EDEC_00_17.pdf. Acesso em 13 de agosto de 2018.

KUMMEROW, C.; SIMPSON, J.; THIELE, O.; BARNES, W.; CHANG, A. T. C.; STOCKER, E.; ADLER, R. F.; HOU, A.; KAKAR, R.; WENTZ, F.; ASHCROFT, P.; KOZU, T.; HONG, Y.; OKAMOTO, K.; IGUCHI, T.; KUROIWA, H.; IM, E.; 
HADDAD, Z.; HUFFMAN, G.; KRISHNAMURTI, T.; FERRIER, B.; OLSON, W. S.; ZIPSER, E.; SMITH, E. A.; WILHEIT, T. T.; NORTH, G.; NAKAMURA, K. The status of the Tropical Rainfall Measuring Mission (TRMM) after two years in orbit, Journal of Applied Meteorology, v. 39, p. 1965-1982, 2000.

LIEBMANN, B.; I. BLADÉ.; G. N. KILADIS.; L. M. CARVALHO.; G. B. SENAY, D.; ALLURED, S.; LEROUX.; C. FUNK. Seasonality of African precipitation from 1996 to 2009, Journal of Climate, v. 25, n. 12, p. 4304-4322, 2012.

MARENGO, J. A. Mudanças Climáticas, Condições Meteorológicas Extremas e Eventos Climáticos no Brasil. FBDS (org.). Mudanças Climáticas e Eventos Extremos no Brasil. FDBS \& LLOYD'S, p. 05-19, 2010.

MELLO, C. R.; SILVA, A. M.; LIMA, J. M.; FERREIRA, D. F.; OLIVEIRA, M. S. Modelos matemáticos para predição da chuva de projeto para regiões do Estado de Minas Gerais, Revista Brasileira de Engenharia Agrícola e Ambiental, v.7, n.1, p.Jan./Abr. 2003.

MINUZZI, R. B. et al. El niño: Ocorrência e duração dos veranicos do estado de Minas Gerais, Revista brasileira de engenharia agrícola e ambiental, v. 9, n. 3, p. 364-371, 2005.

MORAES, B. C.; COSTA, J. M. N.; COSTA, A. C. L.; COSTA, M. H. Variação espacial e temporal da precipitação no estado do Pará, Acta Amazônica, v. 35, n. 2, p. 207-214, 2005.

NOGUEIRA, S. M. C.; MOREIRA, M. A.; VOLPATO, M. M. L. Evaluating Precipitation Estimates from Eta, TRMM and CHRIPS Data in the SouthSoutheast Region of Minas Gerais State-Brazil, Remote Sensing, v. 10, n. 2, p. 313, 2018.

NUNES, L. H.; VICENTE, A. K.; CANDIDO, D. H. Clima da Região Sudeste do Brasil. IN: CAVALCANTI, I. F. de A. et al. (organizadores). Tempo e Clima no Brasil. São Paulo: Oficina de textos, 2009.

PEREIRA, G.; SILVA, M. E. S.; MORAES, E. C.; CARDOZO, F. S. Avaliação dos Dados de Precipitação Estimados pelo Satélite TRMM para o Brasil, Revista Brasileira de Recursos Hídricos, v. 18, n.3, p.139-148, 2013.

PERTUSSATTI, C. A. Veranicos no estado do Mato Grosso: Análise da variabilidade espaço-temporal e modelagem estatística. 2013. 117f. Dissertação (Mestrado em Tecnologia Ambiental e Recursos Hídricos) - Universidade de Brasília, Brasília/DF. 2013.

REBOITA, M. S. et al. Aspectos Climáticos do Estado de Minas Gerais, Revista Brasileira de Climatologia, v. 17, p. 206-226, 2015.

SILVA, E. D. da.; REBOITA, M. S. Estudo da Precipitação no estado de Minas Gerais - MG, Revista Brasileira de Climatologia, v.13, p. 120-136, 2013.

TEO, C.; KOH, T.; CHUN-FUNG LO, J.; CHANDRA BHATT, B. Principal Component Analysis of Observed and Modeled Diurnal Rainfall in the Maritime Continent, J. Climate, v. 24, p. 4662-4675, https://doi.org/10.1175/2011JCLI4047.1, 2011.

TWARDOSZ, R.; CEBULSKA, M.; WALANUS, A. Anomalously heavy monthly and seasonal precipitation in the Polish Carpathian Mountains and their foreland during the years 1881-2010, Theoretical and Applied Climatology, v. 126, p. 1$15,2015$. 
VÁSQUEZ, P. L. M. N.; MOLION, L. C. B.; ARAUJO ABDALAD, M.; MOREIRA, D. M.; SANCHEZ, A. Historical analysis of interannual rainfall variability and trends in southeastern Brazil based on observational and remotely sensed data, Climate Dynamics, v. 50, n. 3, p. 801-824, 2018.

WILKS, D.S. Statistical Methods in the Atmospheric Sciences. 3rd Edition, Academic Press, Oxford, 2011. 704p.

WILSON-DIAZ, D.; MARIANO, A. J.; EVANS, R. H.; LUTHER, M. E. A principal component analysis of sea-surface temperature in the Arabian Sea, Deep Sea Research Part II: Topical Studies in Oceanography, v. 48, Issues 6-7, p. 1097$1114,2001$. 